Original Research Paper

\title{
A Comparative Analysis on the Performance of the Convoluted Exponential Distribution and the Exponential Distribution in terms of Flexibility
}

\author{
${ }^{1}$ Enahoro Alfred Owoloko, ${ }^{1}$ Pelumi Emmanuel Oguntunde and ${ }^{2}$ Adebowale Olusola Adejumo \\ ${ }^{I}$ Department of Mathematics, Covenant University, Ogun State, Nigeria \\ ${ }^{2}$ Department of Statistics, University of Ilorin, Kwara State, Nigeria
}

\author{
Article history \\ Received: 01-09-2015 \\ Revised: $12-04-2016$ \\ Accepted: 15-04-2016 \\ Corresponding Author: \\ Pelumi Emmanuel Oguntunde \\ Department of Mathematics, \\ Covenant University, Ogun \\ State, Nigeria \\ Email: peluemman@yahoo.com
}

\begin{abstract}
In this article, the convoluted exponential distribution which was derived as the sum of two independent exponentially distributed random variables was compared with the exponential distribution in terms of flexibility when applied to four real data sets. The idea is to verify if the convoluted exponential distribution would perform better than the exponential distribution in modeling real life situations. Some other basic statistical properties of the convoluted exponential distribution were also identified.
\end{abstract}

Keywords: Convolution, Distributions, Exponential, Properties

\section{Introduction}

The concept of convolution is a very useful topic in the theory of statistics. As a result, a number of researchers have worked on the sum of Independent and Identically Distributed (IID) random variables. For instance, Sun (2011) defined and studied the convoluted beta Weibull distribution, Shittu et al. (2012) proposed and studied the convoluted beta exponential distribution, Oguntunde et al. (2014) studied the convoluted exponential distribution. Meanwhile, applications to real data sets were not examined in all these researches.

Let $Z$ denote a random variable, it has the Probability Density Function (PDF) of the convoluted beta Weibull distribution (the sum of two independent beta Weibull variates) given by:

$$
f(z)=\frac{\beta_{1} \beta_{2}}{\beta_{1} \gamma_{2}-\beta_{2} \gamma_{1}}\left(e^{-\frac{\beta_{2}}{\gamma_{2}} z}-e^{-\frac{\beta_{1}}{\gamma_{1}} z}\right)
$$

The corresponding Cumulative Density Function (CDF) is given by:

$$
F(z)=\frac{\beta_{2} \gamma_{1}}{\beta_{1} \gamma_{2}-\beta_{2} \gamma_{1}} e^{-\frac{\beta_{1}}{\gamma_{1}} z}-\frac{\beta_{1} \gamma_{2}}{\beta_{1} \gamma_{2}-\beta_{2} \gamma_{1}} e^{-\frac{\beta_{2}}{\gamma_{2}} z}+1
$$

For $z, \beta_{1}, \beta_{2}, \gamma_{1}, \gamma_{2},>0$ and $\beta_{1} \gamma_{2} \neq \beta_{2} \gamma_{1}$ where, $\beta_{1}, \beta_{2}, \gamma_{1}$ and $\gamma_{2}$ are scale parameters.

Details about how Equation 1 and 2 were derived are rigorously explained in Sun (2011).

In the same way, the PDF of the convoluted beta exponential distribution is given by:

$$
f(z)=\frac{\left(b_{1} b_{2} \lambda_{1} \lambda_{2}\right)}{\left(b_{2} \lambda_{2}-b_{1} \lambda_{1}\right)} e^{-b_{2} \lambda_{2} z}\left[1-e^{-\left(b_{2} \lambda_{2}-b_{1} \lambda_{1}\right) z}\right]
$$

The corresponding CDF is given by:

$$
F(z)=\frac{\left(b_{1} b_{2} \lambda_{1} \lambda_{2}\right)}{\left(b_{1} \lambda_{1}-b_{2} \lambda_{2}\right)}\left[\frac{1}{b_{2} \lambda_{2}}\left(1-e^{-b_{2} \lambda_{2} z}\right)-\frac{1}{b_{1} \lambda_{I}}\left(1-e^{-b_{1} \lambda_{1} z}\right)\right]
$$

For $b_{1}, b_{2}, \lambda_{1}, \lambda_{2}, z>0$

For details about the construction of Equation 3 and 4, readers are referred to Shittu et al. (2012).

Another interesting part of the concept of convolution is when the sum of independent random variables from different distributions is considered.

The interest of this research is to further explore the convoluted exponential distribution defined in Oguntunde et al. (2014) by assessing its flexibility over the exponential distribution using four real data sets. The rest of this paper is structured as follows; details about the convoluted exponential distribution (including existing and new properties) are provided in section 2, real life applications are discussed in section 3, followed by a concluding remark.

\section{Convoluted Exponential Distribution: Existing and More Properties}

In this section, the PDF, CDF and basic properties of the convoluted exponential distribution are highlighted as available in Oguntunde et al. (2014). Also, some other new properties are given. 
The PDF of the convoluted exponential distribution is given by:

$$
f(z)=\frac{\lambda_{1} \lambda_{2}}{\lambda_{2}-\lambda_{1}}\left(e^{-\lambda_{1} z}-e^{-\lambda_{2} z}\right)
$$

The corresponding CDF is given by:

$$
F(z)=1+\frac{\lambda_{1}}{\lambda_{2}-\lambda_{1}} e^{-\lambda_{2} z}-\frac{\lambda_{2}}{\lambda_{2}-\lambda_{1}} e^{-\lambda_{1} z}
$$

For $\lambda_{1}, \lambda_{2}, z>0$

where; $\lambda_{1}$ and $\lambda_{2}$ are scale parameters

The mean is given by:

$$
\begin{aligned}
& E(Z)=\frac{1}{\lambda_{1}}+\frac{1}{\lambda_{2}} \\
& \operatorname{Var}(Z)=\frac{1}{\lambda_{1}^{2}}+\frac{1}{\lambda_{2}^{2}} \\
& \text { Skewness }=\frac{2\left[\left(\frac{1}{\lambda_{1}}\right)^{3}+\left(\frac{1}{\lambda_{2}}\right)^{3}\right]}{\left[\left(\frac{1}{\lambda_{1}}\right)^{2}+\left(\frac{1}{\lambda_{2}}\right)^{2}\right]^{3 / 2}} \\
& \text { Kurtosis }=\frac{6\left[\left(\frac{1}{\lambda_{1}}\right)^{4}+\left(\frac{1}{\lambda_{2}}\right)^{4}\right]}{\left[\left(\frac{1}{\lambda_{1}}\right)^{2}+\left(\frac{1}{\lambda_{2}}\right)^{2}\right]^{2}}
\end{aligned}
$$

The moment generating function is given by:

$$
M_{Z}(t)=\frac{\lambda_{1} \lambda_{2}}{\left(\lambda_{1}-t\right)\left(\lambda_{2}-t\right)}
$$

\section{Renyi Entropy}

The Renyi entropy being one of the functions used in quantifying the uncertainty or randomness in a system is mathematically given by:

$$
R(p)=\frac{1}{1-p} \log \left[\int_{-\infty}^{\infty} f^{p}(z) d z\right]
$$

For $p \neq 1$ and $p>0$

For the Convoluted Exponential distribution, the entropy is derived from:

$$
\begin{aligned}
& R(p)=\frac{1}{1-p} \log \left[\int_{0}^{\infty}\left\{\frac{\lambda_{1} \lambda_{2}}{\lambda_{2}-\lambda_{1}}\left(e^{-\lambda_{1} z}-e^{-\lambda_{2} z}\right)\right\}^{p} d z\right] \\
& =\frac{1}{1-p} \log \left[\int_{0}^{\infty} \frac{\lambda_{1}^{p} \lambda_{2}^{p}}{\left(\lambda_{2}-\lambda_{1}\right)^{p}}\left(e^{-\lambda_{1} z}-e^{-\lambda_{2} z}\right)^{p} d z\right] \\
& =\frac{1}{1-p} \log \left[\frac{\lambda_{1}^{p} \lambda_{2}^{p}}{\left(\lambda_{2}-\lambda_{1}\right)^{p}} \int_{0}^{\infty}\left(e^{-\lambda_{1} z}-e^{-\lambda_{2} z}\right)^{p} d z\right] \\
& =\frac{1}{1-p}\left\{\log \left[\frac{\lambda_{1}^{p} \lambda_{2}^{p}}{\left(\lambda_{2}-\lambda_{1}\right)^{p}}\right]+\left[\log \int_{0}^{\infty}\left(e^{-\lambda_{1} z}-e^{-\lambda_{2} z}\right)^{p} d z\right]\right\} \\
& =\frac{1}{1-p}\left\{\log \left(\frac{\lambda_{1} \lambda_{2}}{\lambda_{2}-\lambda_{1}}\right)^{p}+\log \int_{0}^{\infty}\left(e^{-\lambda_{1} z}-e^{-\lambda_{2} z}\right)^{p} d z\right\} \\
& =\frac{1}{1-p}\left\{p \log \left(\frac{\lambda_{1} \lambda_{2}}{\lambda_{2}-\lambda_{1}}\right)+\log \left[\int_{0}^{\infty} \sum_{i=0}^{p}\left(\begin{array}{l}
p \\
i
\end{array}\right)(-1)^{i}\left(e^{-\lambda_{1} z}\right)^{p-1}\left(e^{-\lambda_{2} z}\right)^{i} d z\right]\right\} \\
& =\frac{1}{1-p}\left\{p \log \left(\frac{\lambda_{1} \lambda_{2}}{\lambda_{2}-\lambda_{1}}\right)+\log \left[\sum_{i=0}^{p}(i) \int_{0}^{\infty}(-1)^{i}\left(e^{-\lambda_{1} z(p-i)}\right)\left(e^{-\lambda_{2} z i}\right) d z\right]\right\} \\
& =\frac{1}{1-p}\left\{p \log \left(\frac{\lambda_{1} \lambda_{2}}{\lambda_{2}-\lambda_{1}}\right)+\log \left[\sum_{i=0}^{p}\left(\begin{array}{l}
p \\
i
\end{array} \int_{0}^{\infty}(-1)^{i}\left(e^{-\left(\lambda_{1}(p-i)+\lambda_{2} i\right) z}\right) d z\right]\right\}\right. \\
& =\frac{1}{1-p}\left\{p \log \left(\frac{\lambda_{1} \lambda_{2}}{\lambda_{2}-\lambda_{1}}\right)+\log \sum_{i=0}^{p}\left(\begin{array}{l}
p \\
i
\end{array}\right)\left[(-1)^{i+1} \frac{e^{-\left(\lambda_{1}(p-i)+\lambda_{2} i\right) z}}{\lambda_{1} p-\lambda_{1} i+\lambda_{2} i}\right]_{0}^{\infty}\right\} \\
& =\frac{1}{1-p}\left\{p \log \left(\frac{\lambda_{1} \lambda_{2}}{\lambda_{2}-\lambda_{1}}\right)+\log \left[\sum_{i=0}^{p}\left(\begin{array}{l}
p \\
i
\end{array}\right)(-1)^{i+1} \frac{1}{\lambda_{1} p-\lambda_{1} i+\lambda_{2} i}(0-1)\right]\right\} \\
& =\frac{1}{1-p}\left\{p \log \left(\frac{\lambda_{1} \lambda_{2}}{\lambda_{2}-\lambda_{1}}\right)+\log \left[\sum_{i=0}^{p}\left(\begin{array}{l}
p \\
i
\end{array}\right)(-1)^{i} \frac{1}{\lambda_{1} p+\left(\lambda_{2}-\lambda_{1}\right) i}\right]\right\} ; p \neq 1
\end{aligned}
$$

\section{Emperical Study}

The tables for the mean and variance of the convoluted exponential distribution are provided in Table 1 and 2 respectively.

It can be observed from Table 1 that the mean of the Convoluted Exponential distribution decreases as the parameter increases and vice versa.

Table 2 reveals that the variance of the Convoluted Exponential distribution decreases as the value of the parameters increases and vice versa.

\section{Application}

The convoluted exponential distribution and the exponential distribution are both applied to four real data sets and the distribution corresponding to the best fit is selected using the Akaike Information Criteria (AIC) and the Log-likelihood. 
Table 1. Mean of the convoluted exponential distribution at different parameter values

\begin{tabular}{lllllllllll}
\hline & $\lambda_{2}=1$ & $\lambda_{2}=2$ & $\lambda_{2}=3$ & $\lambda_{2}=4$ & $\lambda_{2}=5$ & $\lambda_{2}=6$ & $\lambda_{2}=7$ & $\lambda_{2}=8$ & $\lambda_{2}=9$ & $\lambda_{2}=10$ \\
\hline$\lambda_{1}=1$ & 2.000 & 1.500 & 1.333 & 1.250 & 1.200 & 1.167 & 1.143 & 1.125 & 1.111 & 1.100 \\
$\lambda_{1}=2$ & 1.500 & 1.000 & 0.833 & 0.750 & 0.700 & 0.667 & 0.643 & 0.625 & 0.611 & 0.600 \\
$\lambda_{1}=3$ & 1.333 & 0.833 & 0.667 & 0.583 & 0.533 & 0.500 & 0.476 & 0.458 & 0.444 & 0.433 \\
$\lambda_{1}=4$ & 1.250 & 0.750 & 0.583 & 0.500 & 0.450 & 0.417 & 0.393 & 0.375 & 0.361 & 0.350 \\
$\lambda_{1}=5$ & 1.200 & 0.700 & 0.533 & 0.450 & 0.400 & 0.367 & 0.343 & 0.325 & 0.311 & 0.300 \\
$\lambda_{1}=6$ & 1.167 & 0.67 & 0.500 & 0.417 & 0.367 & 0.333 & 0.310 & 0.292 & 0.278 & 0.267 \\
$\lambda_{1}=7$ & 1.143 & 0.643 & 0.476 & 0.393 & 0.343 & 0.310 & 0.286 & 0.268 & 0.254 & 0.243 \\
$\lambda_{1}=8$ & 1.125 & 0.625 & 0.458 & 0.375 & 0.325 & 0.292 & 0.268 & 0.250 & 0.236 & 0.225 \\
$\lambda_{1}=9$ & 1.111 & 0.611 & 0.444 & 0.361 & 0.311 & 0.278 & 0.254 & 0.236 & 0.222 & 0.211 \\
$\lambda_{1}=10$ & 1.100 & 0.600 & 0.433 & 0.350 & 0.300 & 0.267 & 0.243 & 0.225 & 0.211 & 0.200 \\
\hline
\end{tabular}

Table 2. Variance of the convoluted exponential distribution at different parameter values

\begin{tabular}{|c|c|c|c|c|c|c|c|c|c|c|}
\hline & $\lambda_{2}=1$ & $\lambda_{2}=2$ & $\lambda_{2}=3$ & $\lambda_{2}=4$ & $\lambda_{2}=5$ & $\lambda_{2}=6$ & $\lambda_{2}=7$ & $\lambda_{2}=8$ & $\lambda_{2}=9$ & $\lambda_{2}=10$ \\
\hline$\lambda_{1}=1$ & 2.000 & 1.250 & 1.111 & 1.062 & 1.040 & 1.028 & 1.020 & 1.016 & 1.012 & 1.010 \\
\hline$\lambda_{1}=2$ & 1.250 & 0.500 & 0.361 & 0.313 & 0.290 & 0.278 & 0.270 & 0.266 & 0.262 & 0.260 \\
\hline$\lambda_{1}=3$ & 1.111 & 0.361 & 0.222 & 0.174 & 0.151 & 0.139 & 0.132 & 0.127 & 0.123 & 0.121 \\
\hline$\lambda_{1}=4$ & 1.063 & 0.313 & 0.174 & 0.125 & 0.103 & 0.090 & 0.083 & 0.078 & 0.075 & 0.073 \\
\hline$\lambda_{1}=5$ & 1.040 & 0.290 & 0.151 & 0.103 & 0.080 & 0.068 & 0.060 & 0.056 & 0.052 & 0.050 \\
\hline$\lambda_{1}=6$ & 1.028 & 0.278 & 0.139 & 0.090 & 0.068 & 0.056 & 0.048 & 0.043 & 0.040 & 0.038 \\
\hline$\lambda_{1}=7$ & 1.020 & 0.270 & 0.132 & 0.083 & 0.060 & 0.048 & 0.041 & 0.036 & 0.033 & 0.030 \\
\hline$\lambda_{1}=8$ & 1.016 & 0.266 & 0.127 & 0.078 & 0.056 & 0.043 & 0.036 & 0.031 & 0.028 & 0.026 \\
\hline$\lambda_{1}=9$ & 1.012 & 0.262 & 0.123 & 0.075 & 0.052 & 0.040 & 0.033 & 0.028 & 0.025 & 0.022 \\
\hline$\lambda_{1}=10$ & 1.010 & 0.260 & 0.121 & 0.073 & 0.050 & 0.038 & 0.030 & 0.026 & 0.022 & 0.020 \\
\hline
\end{tabular}

\section{Data Set I}

The first data set represents the height of 100 female athletes (measured in centimeters); it is one of the thirteen variables in the Australian athletes' data reported in Cook and Weisberg (1994). The data was collected at the Australian Institute of Sport. It has been previously used and analyzed by (Jamalizadeh et al., 2011; Choudhury and Abdul Matin, 2011; Al-Aqtash et al., 2014). The data is as follows:

$148.9,149.0,156.0,156.9,157.9,158.9,162.0,162.0$, $162.5,163.0,163.9,165.0,166.1,166.7,167.3,167.9$, $168.0,168.6,169.1,169.8,169.9,170.0,170.0,170.3$, $170.8,171.1,171.4,171.4,171.6,171.7,172.0,172.2$, $172.3,172.5,172.6,172.7,173.0,173.3,173.3,173.5$, $173.6,173.7,173.8,174.0,174.0,174.0,174.1,174.1$, $174.4,175.0,175.0,175.0,175.3,175.6,176.0,176.0$, $176.0,176.0,176.8,177.0,177.3,177.3,177.5,177.5$, $177.8,177.9,178.0,178.2,178.7,178.9,179.3,179.5$, $179.6,179.6,179.7,179.7,179.8,179.9,180.2,180.2$, $180.5,180.5,180.9,181.0,181.3,182.1,182.7,183.0$, $183.3,183.3,184.6,184.7,185.0,185.2,186.2,186.3$, 188.7, 189.7, 193.4, 195.9 .

The data is summarized in Table 3 and the performances of the competing distributions are given in Table 4.

\section{Data Set II}

The second data set was reported by (Bjerkedal, 1960) and it has also been studied by (Tahir et al., 2014). It represents the survival times of 72 guinea pigs (in days) infected with virulent tubercle. The data is as follows:

$10,33,44,56,59,72,74,77,92,93,96,100,100,102$, $105,107,107,108,108,108,109,112,113,115,116$, $120,121,122,122,124,130,134,136,139,144,146$, $153,159,160,163,163,168,171,172,176,183,195$, $196,197,202,213,215,216,222,230,231,240,245$, $251,253,254,255,278,293,327,342,347,361,402$, $432,458,555$.

The data is summarized in Table 5 and the performances of the competing distributions are given in Table 6.

\section{Data Set III}

The third data set has been previously studied by (Quesenberry and Kent, 1982; Pal and Tiensuwan, 2014). It represents the time to failure of a ployster/viscose yarn in a textile experiment at $2.3 \%$ strain level. The data is as follows:

$86,146,251,653,98,249,400,292,131,169$, $175,176,76,264,15,364,195,262,88,264,157$, 
$220,42,321,180,198,38,20,61,121,282,224,149$, $180,325,250,196,90,229,166,38,337,65,151$, $341,40,40,135,597,246,211,180,93,315,353$, $571,124,279,81,186,497,182,423,185,229,400$, $338,290,398,71,246,185,188,568,55,55,61,244$,
$20,284,393,396,203,829,239,236,286,194,277$, $143,198,264,105,203,124,137,135,350,193,188$.

The data is summarized in Table 7 and the performances of the competing distributions are given in Table 8.

Table 3. Summary of data on height of 100 female athletes

\begin{tabular}{lllllllll}
\hline Min. & Q1 & Q2 & Q3 & Mean & Variance & Skewness & Kurtosis & Max. \\
\hline 148.9 & 171.0 & 175.0 & 179.7 & 174.6 & 67.9339 & -0.5598 & 4.1967 & 195.9 \\
\hline
\end{tabular}

Table 4. Performance of distributions with standard errors in parentheses using DATA I

\begin{tabular}{lllll}
\hline Distributions & Estimates & t-statistic & p-value & \multicolumn{2}{c}{ Log-likelihood AIC } \\
Exponential & $\hat{\lambda}=0.0057276(0.0005729)$ & 9.998 & $<2 \times 10^{-16}$ & -616.2463 \\
Convoluted exponential & $\hat{\lambda}_{1}=0.005728(0.0005729)$ & 9.998 & $<2 \times 10^{-16}$ & -616.2449 \\
& $\hat{\lambda}_{2}=391.7(2.966)$ & 132.07 & $<2 \times 10^{-16}$ & 1236.493 \\
& & & \\
\hline
\end{tabular}

Table 5. Summary of data on survival times of 72 guinea pigs

\begin{tabular}{lllllllll}
\hline Min. & Q1 & Q2 & Q3 & Mean & Variance & Skewness & Kurtosis & Max. \\
\hline 10.0 & 108.0 & 149.5 & 224.0 & 176.8 & 10705.1 & 1.3413 & 4.9885 & 555.0 \\
\hline
\end{tabular}

Table 6. Performance of distributions with standard errors in parentheses using DATA II

\begin{tabular}{llllr}
\hline Distributions & Estimates & t-statistic & p-value & \multicolumn{2}{l}{ Log-likelihood AIC } \\
\hline Exponential & $\hat{\lambda}=0.0056550(0.0006666)$ & 8.484 & $<2 \times 10^{-16}$ & -444.615 \\
Convoluted exponential & $\hat{\lambda}_{1}=0.005655(0.0006666)$ & 8.484 & $<2 \times 10^{-16}$ & -444.614 \\
& $\hat{\lambda}_{2}=0.04461(2.966)$ & 150.429 & $<2 \times 10^{-16}$ & 893.2281 \\
& &
\end{tabular}

Table 7. Summary of data on failure times of Yarn at $2.3 \%$ strain level

\begin{tabular}{lllllllll}
\hline Min. & Q1 & Q2 & Q3 & Mean & Variance & Skewness & Kurtosis & Max. \\
\hline 15.0 & 129.2 & 195.5 & 282.5 & 222.0 & 20914.38 & 1.3600 & 5.8601 & 829.0 \\
\hline
\end{tabular}

Table 8. Performance of distributions with standard errors in parentheses using DATA III

\begin{tabular}{|c|c|c|c|c|c|}
\hline Distributions & Estimates & t-statistic & $\mathrm{p}$-value & Log-likelihood & AIC \\
\hline Exponential & $\hat{\lambda}=0.0045049(0.0004506)$ & 9.988 & $<2 \times 10^{-16}$ & -640.2587 & 1282.517 \\
\hline \multirow[t]{2}{*}{ Convoluted exponential } & $\hat{\lambda}_{1}=0.004505(0.0004506)$ & 9.998 & $<2 \times 10^{-16}$ & -640.2585 & 1284.517 \\
\hline & $\hat{\lambda}_{2}=2309(2.966)$ & 778.597 & $<2 \times 10^{-16}$ & & \\
\hline
\end{tabular}

Table 9. Summary of data on electronic components

\begin{tabular}{lllllllll}
\hline Min. & Q1 & Q2 & Q3 & Mean & Variance & Skewness & Kurtosis & Max. \\
\hline 0.030 & 0.775 & 1.795 & 2.900 & 1.936 & 2.062 & 0.603 & 2.720 & 5.090 \\
\hline
\end{tabular}

Table 10. Performance of distributions with standard errors in parentheses using DATA IV

\begin{tabular}{llllll}
\hline Distributions & Estimates & t-statistic & p-value & Log-likelihood & AIC \\
\hline Exponential & $\hat{\lambda}=0.5167(0.1155)$ & 4.472 & $7.75 \times 10^{-6}$ & -33.20731 & 68.41463 \\
Convoluted exponential & $\hat{\lambda}_{1}=0.5187(0.1160)$ & 4.471 & $7.78 \times 10^{-6}$ & -33.14827 & 70.29655 \\
& $\hat{\lambda}_{2}=131.4146(8.3888)$ & 15.665 & $<2 \times 10^{-16}$ & & \\
\hline
\end{tabular}




\section{Data Set IV}

The fourth data set has been previously used by (Teimouri and Gupta, 2013; Nasiru, 2015). It represents the lifetime of 20 electronic components. The data is as follows:

$0.03,0.22,0.73,1.25,1.52,1.8,2.38,2.87,3.14,4.72$, $0.12,0.35,0.79,1.41,1.79,1.94,2.4,2.99,3.17,5.09$

The data is summarized in Table 9 and the performances of the competing distributions are given in Table 10.

\section{Remark}

Considering Table 4, 6, 8 and 10, the model with the lowest AIC or highest log-likelihood is considered to be the best fit. This means that the exponential distribution is considered the best fit and thereby highlighted.

\section{Conclusion}

A comparison between the convoluted exponential distribution and the exponential distribution has been successfully done in terms of real life applications. It was observed that the exponential distribution outperformed the convoluted exponential distribution considering the four applications provided in this research. The decisions and conclusion in this study is based on the log-likelihood and AIC values posed by the distributions under study. For all the four data sets, the AIC value of the Exponential distribution is the lowest while its log-likelihood values are higher than that of the Convoluted Exponential distribution. Nevertheless, the authors did not underrate the concept of convolution. Convolution still remains a relevant topic in the theory of statistics. Further research would involve comparing convoluted beta Weibull distribution derived by (Sun, 2011) with beta Weibull distribution derived by (Famoye et al., 2005) and comparing convoluted beta exponential distribution derived by (Shittu et al., 2012) with beta exponential distribution derived by (Nadarajah and Kotz, 2006) to assess their flexibilities in modeling real life data sets.

\section{Acknowledgement}

The authors appreciate the effort of the anonymous reviewers for their timely and useful comments. The financial support from Covenant University is also acknowledged.

\section{Author's Contributions}

Enahoro Alfred Owoloko: Being a Co-supervisor, he read through the manuscript, reviewed, corrected and approved it before sending to a journal outlet.
Pelumi Emmanuel Oguntunde: Being a research student, he conceived the idea and drafted the paper.

Adejumo Olusola Adejumo: Being a supervisor, he guided the student in the write-up, checked the analysis and codes used for correctness.

\section{Ethics}

This article is original and contains unpublished material. The corresponding author confirms that all of the other authors have read and approved the manuscript and no ethical issues involved.

\section{References}

Al-Aqtash, R., C. Lee and F. Famoye, 2014. Gumbelweibull distribution: Properties and applications. J. Modern Applied Stat. Meth, 13: 201-225.

Bjerkedal, T., 1960. Acquisition of resistance in guinea pigs infected with different doses of virulent tubercle bacilli. Am. J. Hygiene, 72: 130-148. PMID: 13801076

Choudhury, K. and M. Abdul Matin, 2011. Extended skew generalized normal distribution. Metron, 69: 265-278. DOI: 10.1007/BF03263561

Cook, R.D. and S. Weisberg, 1994. An Introduction to Regression Graphics. 1st Edn., John Wiley and Sons, New York, ISBN-10: 0470317701, pp: 280.

Famoye, F., C. Lee and O. Olugbenga, 2005. The beta-weibull distribution. J. Stat. Theory Applic., 4: 121-138.

Jamalizadeh, A., A.R. Arabpour and N. Balakrishnan, 2011. A generalized skew two-piece skew-normal distribution. Stat. Papers, 52: 431-446. DOI: $10.1007 / \mathrm{s} 00362-009-0240-x$

Nadarajah, S. and S. Kotz, 2006. The beta exponential distribution. Reliab. Eng. Syst. Safety, 91: 689-697. DOI: 10.1016/j.ress.2005.05.008

Nasiru, S., 2015. Another weighted Weibull distribution from azzalini's family. Eur. Sci. J., 11: 134-144.

Oguntunde, P.E., O.A. Odetunmibi and A.O. Adejumo, 2014. On the sum of exponentially distributed random variables: A convolution approach. Eur. J. Stat. Probab., 2: 1-8. DOI: 10.1155/2014/864965

Pal, M. and M. Tiensuwan, 2014. The beta transmuted weibull distribution. Aus. J. Stat., 43: 133-149. DOI: 10.17713 /ajs.v43i2.37

Quesenberry, C.P. and J. Kent, 1982. Selecting among probability distributions used in reliability. Technometrics, 24: 59-65. DOI: $10.1080 / 00401706.1982 .10487710$

Shittu, O.I., K.A. Adepoju and O.S. Yaya, 2012. On the convoluted beta-exponential distribution. J. Modern Math. Stat., 6: 14-22.

DOI: $10.3923 /$ jmmstat.2012.14.22 
Sun, J., 2011. Statistical properties of a convoluted betaWeibull distribution. MSc Thesis, Marshall University.

Tahir, M.H., G.M. Cordeiro, A. Alzaatreh and M. Mansoor, 2014. The logistic-x family of distributions and its applications. Commun. Statist Theory Meth.

DOI: 10.1080/03610926.2014.980516
Teimouri, M. and K.A. Gupta, 2013. On three parameter weibull distribution shape parameter estimation. J. Data Sci., 11: 403-414. 